\title{
AVALIAÇÃO DA CAPACIDADE DE ADSORÇÃO DE SEMENTE DE Moringa oleifera LAM PARA REMOÇÃO DO HERBICIDA ATRAZINA EM AMOSTRAS AQUOSAS
}

\author{
P. F. COLDEBELLA ${ }^{1}$, K. C. VALVERDE ${ }^{1}$, F. G. BORTOLOZZO ${ }^{1}$, L. NISHI ${ }^{1}$, D. REZENDE ${ }^{1}$, M. \\ F. SILVA ${ }^{1}$, M. R. FAGUNDES-KLEN ${ }^{2}$, A. M. S. VIEIRA ${ }^{1}$, M. F. VIEIRA ${ }^{1}$, O. A. A. SANTOS ${ }^{1}$ e R. \\ BERGAMASCO $^{1}$
}

${ }^{1}$ Universidade Estadual de Maringá, Departamento de Engenharia Química e Engenharia de Alimentos

${ }^{2}$ Universidade Estadual do Oeste do Paraná, Departamento de Engenharia Química E-mail para contato: priscila.ferri@bol.com.br

\begin{abstract}
RESUMO - As sementes de Moringa oleifera Lam (MO) tem sido utilizado como um biossorvente barato e eficaz para a remoção de contaminantes orgânicos. Neste estudo a capacidade de adsorção de Atrazina (ATZ) em amostras aquosas foi avaliada. A MO foi separada das cascas, triturada e separada em na granulometria de $500 \mu \mathrm{m}$. A MO foi caracterizada por espectroscopia de infravermelho, difratometria de raios $\mathrm{X}$, microscopia eletrônica de varredura e ponto de carga zero. Uma solução de $5 \mathrm{mg} \mathrm{L}^{1}{ }^{1}$ de atrazina foi preparada e analisada por HPLC. Avaliou-se o efeito de diferentes parâmetros, velocidade de agitação $100-200 \mathrm{rpm}, \mathrm{pH} 2-10$, temperatura $25-45^{\circ} \mathrm{C}$, a dose de adsorvente $0,05-1,2 \mathrm{~g}$, tempo de contato 2-240 min,, e concentração de sorbato $0,1-15 \mathrm{mg} \mathrm{L}-1$, sobre o potencial de sorção da Atrazina. Isotermas de adsorção Langmuir, Freundlich e modelos cinéticos pseudo primeira e segunda ordem foram empregados. Os resultados de adsorção de $78 \%$ de remoção de atrazina foram alcançados para $\mathrm{q}_{\mathrm{eq} \text { experimental }}=0,352 \mathrm{mg} \mathrm{g}^{-1}$, na velocidade de agitação de $100 \mathrm{rpm}, \mathrm{pH} 4$, temperatura $25^{\circ} \mathrm{C}$ e tempo de equilíbrio de 20 minutos. Baseando-se nos valores do coeficiente de correlação $\left(\mathrm{r}^{2}=0,971\right)$, a isoterma de Langmuir foi a que apresentou melhor ajuste, com capacidade máxima de adsorção $\left(q_{\text {máx }}\right)$ igual a $0,578 \mathrm{mg} \mathrm{g}^{-1}$. O modelo cinético de pseudo-primeira-ordem foi o que melhor se ajustou aos dados experimentais. Através dos resultados obtidos a MO apresentou como um biossorvente em potencial para remoção de Atrazina.
\end{abstract}

\section{INTRODUÇÃO}

As águas superficiais contêm diferentes compostos que devem ser reduzidos durante o processo de tratamento de água. Entre os compostos que são contaminantes, não biodegradáveis e prejudiciais a saúde humana está os pesticidas. O herbicida Atrazina (ATZ) [2-cloro-4-etilamino6-isopropilamino-s triazina] é um dos pesticidas potencialmente impactantes do meio ambiente 


\section{9 a 22 de outubro de 2014 \\ Florianópolis/SC}

(Ávila, 2009). Esse herbicida é largamente utilizado no Brasil no controle de ervas daninhas e gramíneas, caracterizando-se como medianamente tóxico e de difícil degradação.

Os limites máximos permitidos para a atrazina em águas para consumo humano são de 2 ug. $\mathrm{L}^{-1}, 3 \mu \mathrm{g} . \mathrm{L}^{-1}$ nos Estados Unidos e 0,5 $\mu \mathrm{g} . \mathrm{L}^{-1}$ na Europa (Brasil, 2011; US EPA, 1985; EUROPEAN COMMISSION, 2008), no qual, o processo convencional de tratamento de água não é um suficiente para a remoção de concentrações baixas na ordem de partes por bilhão de pesticidas, tornando necessários tratamentos alternativos. A biossorção pode ser considerada uma alternativa, na qual é o processo de absorção e/ou adsorção passivo de substâncias tóxicas por materiais biológicos inativos ou por materiais derivados de fontes biológicas.

A planta Moringa oleifera (MO) é uma espécie arbórea originária do noroeste indiano, cultivada graças ao seu valor alimentar e medicinal (Bezerra et al., 2004). O seu crescimento é rápido mesmo em solos com pouca umidade (Kwaambwa e Maikokera, 2008). Ela tem sido estudada no tratamento de água no processo de coagulação-floculação e também como biossorvente. Tradicionalmente, em pequenas comunidades rurais, a polpa da semente da $M$. oleifera, ou "Lírio Branco" como é conhecido, é utilizada para remover a turvação de águas para consumo devido a presença de polieletrólitos catiônicos (coagulação-floculação) (Okuda et al., 2001). Existem vários estudos que demonstram a sua capacidade adsortiva de metais (Araujo et al., 2010; Reddy et al., 2011; Alves e Coelho, 2013), alguns compostos orgânicos como Benzeno, Tolueno, Etilbenzeno e Xilenos, BTEX (Almeida, 2010) e Trialometanos, THMs (Cunha et al. (2011).

Nesse sentido o objetivo do trabalho foi de avaliar a capacidade da semente de Moringa oleifera Lam no processo de biossorção do herbicida Atrazina em soluções aquosas.

\section{EXPERIMENTAL}

A amostra em estudo foi soluções contaminadas com atrazina $\left(5 \mathrm{mg} \mathrm{L}^{-1}\right)$ preparadas a partir do produto comercial Atrazina $500 \mathrm{SC}$ Nortox $\left(500 \mathrm{~g} \mathrm{~L}^{-1}\right)$. As soluções de Atrazina foram analisada através de cromatografia líquida de alta eficiência (HPLC) usando um cromatógrafo 920 LC-VIS (Varian, Austrália) de acordo com metodologia proposta por Bortoluzzi et al. (2010).

As sementes de Moringa oleifera Lam provenientes de Aracajú, Sergipe, Brasil foram utilizadas como biossorvente. As sementes foram separadas das cascas e secas em estufa com circulação de ar (Temporizador Digital SX CR/42) à $40^{\circ} \mathrm{C}$ até peso constante (Amagloh e Benang, 2009). As sementes foram triturados e peneirado em diferentes tamanhos de malha 150$700 \mu \mathrm{m}$ em peneiras série agitador tipo magnético (Bertel). O tamanho médio de partículas escolhido para o presente trabalho foi de $500 \mu \mathrm{m}$. As sementes de Moringa oleifera foram caracterizadas quanto a presença de grupos funcionais por Espectroscopia no Infravermelho com Transforma de Fourier (FTIR) Spectrum 100 (PerkinElmer) na faixa de 4000 a $400 \mathrm{~cm}^{-1}$. Para avaliação da presença de fases cristalinas e/ou amorfas nos materiais de estudo, foram obtidos 
difratogramas de raios-x (DRX) utilizando um difratômetro D8-Advance (Bruker). Na avaliação das características morfológicas da semente de Moringa oleifera um microscópio eletrônico de varredura (MEV) SS 550 Superscan (Shimadzu) foi utilizado. O ponto de carga zero (PCZ) foi determinado pelo método "experimento dos 11 pontos" (Regalbuto e Robles, 2004).

O estudo de biossorção foi realizado conforme metodologia de Akhtar et al. (2007). Foram avaliados os parâmetros velocidade de agitação (100-200rpm), efeito do pH (2-10), efeito da temperatura $\left(25-45^{\circ} \mathrm{C}\right)$, massa de adsorvente $(0,05-1,2 \mathrm{~g})$, fixando a concentração de ATZ de 5 $\mathrm{mg} . \mathrm{L}^{-1}$, um volume de solução de $25 \mathrm{ml}$ e o tempo de contato 1 hora. $\mathrm{O} \mathrm{pH}$ foi ajustado com soluções de $\mathrm{NaOH}$ e $\mathrm{HCl}$ 0,1 mol.L $\mathrm{L}^{-1}$. A temperatura e agitação foram controlados em incubadora com agitação orbital (Marconi - MA 420). As amostras foram colhidas e filtradas em filtro de acetato de celulose (Millipore) de 0,45 $\mu \mathrm{m}$ e analisadas em HPLC. A Análise de Variância (ANOVA) e o teste de comparação de médias, teste Tukey, com $95 \%$ de confiança, sendo significativo um p-valor $<0,05$ foi utilizado através do programa estatístico Statistica versão 8.0. $\mathrm{O}$ experimento foi realizado em triplicata.

Para avaliar a cinética de adsorção foi realizado utilizando-se $25 \mathrm{ml}$ de solução de atrazina $5 \mathrm{mg} . \mathrm{L}^{-1}$, massa de $0,6 \mathrm{~g}$ do adsorvente, temperatura à $25^{\circ} \mathrm{C} \pm 2^{\circ} \mathrm{C}, \mathrm{pH} 4 \pm 0,2$ e agitação 100 rpm. As amostras foram coletadas em intervalos de tempo pré-determinados (2 a $240 \mathrm{~min}$ ), filtradas e analisadas em HPLC. A quantidade de Atrazina adsorvida foi determinada pela Equação 1.

$$
q_{e q}=\frac{V\left(C_{0}-C_{e q}\right)}{m}
$$

Em que $q_{e q}$ é a concentração de equilíbrio do atrazina no equilibrio $\left(\mathrm{mg} \mathrm{g}^{-1}\right), V$ o volume de solução $(\mathrm{mL}) ; C_{0}$ a concentração inicial de atrazina na solução $\left(\mathrm{mg} \mathrm{L}^{-1}\right) ; C_{\in q}$ a concentração do atrazina no equilíbrio $\left(\mathrm{mg} \mathrm{L}^{-1}\right)$ e $m$ a massa de adsorvente $(\mathrm{g})$.

Aos dados experimentais foram ajustados os modelos cinéticos de pseudo-primeira e pseudo-segunda ordens, representados pelas Equações 2 e 3, respectivamente.

$$
\begin{aligned}
& q_{t}=q_{e q}\left(1-\exp \left(-k_{p l} t\right)\right) \\
& q_{t}=\frac{k_{p s} q_{e q}^{2} t}{1+k_{p s} q_{e q} t}
\end{aligned}
$$

Em que, $q_{t}$ é a concentração de atrazina na fase sólida no tempo $t\left(\mathrm{mg} \mathrm{g}^{-1}\right)$ e $k_{p l}$ a constante da taxa de adsorção do modelo pseudo-primeira ordem $\left(\min ^{-1}\right)$ e $k_{p s}$ é a constante da taxa de adsorção do modelo pseudo-segunda ordem $\left(\mathrm{g} \mathrm{mg}^{-1} \mathrm{~min}^{-1}\right)$.

Para avaliar o equilíbrio de adsorção variou-se a concentração de atrazina de 0,1 a 15 mg. $L^{-}$ ${ }^{1}$. Foram mantidos constantes a massa de adsorvente $0,6 \mathrm{~g}, \grave{a} 25^{\circ} \mathrm{C} \pm 2{ }^{\circ} \mathrm{C}, \mathrm{pH} 4 \pm 0,2$ e agitação $100 \mathrm{rpm}$ por um período de $1 \mathrm{~h}$. As amostras foram coletadas, filtradas e analisadas por HPLC. 
Aos dados experimentais de equilíbrio foram ajustados aos modelos de isotermas de Langmuir e Freundlich de acordo com Akhtar et al. (2007), onde foram calculados: $q_{\text {máx }}$, a capacidade máxima de adsorção do adsorvente $\left(\mathrm{mg} \cdot \mathrm{g}^{-1}\right), b_{L}$, a constante de afinidade de Langmuir $\left(\mathrm{L} \mathrm{mg}^{-1}\right)$, $k_{F}$ e $n_{F}$ constantes de Freundlich.

\section{RESULTADOS E DISCUSSÃO}

A semente de Moringa oleifera foi caracterizada pelas técnicas FTIR, DRX, MEV e PCZ. Esses resultados são apresentados nas figuras 1 (A), 1 (B), 2 (A e B) e 3 respectivamente.
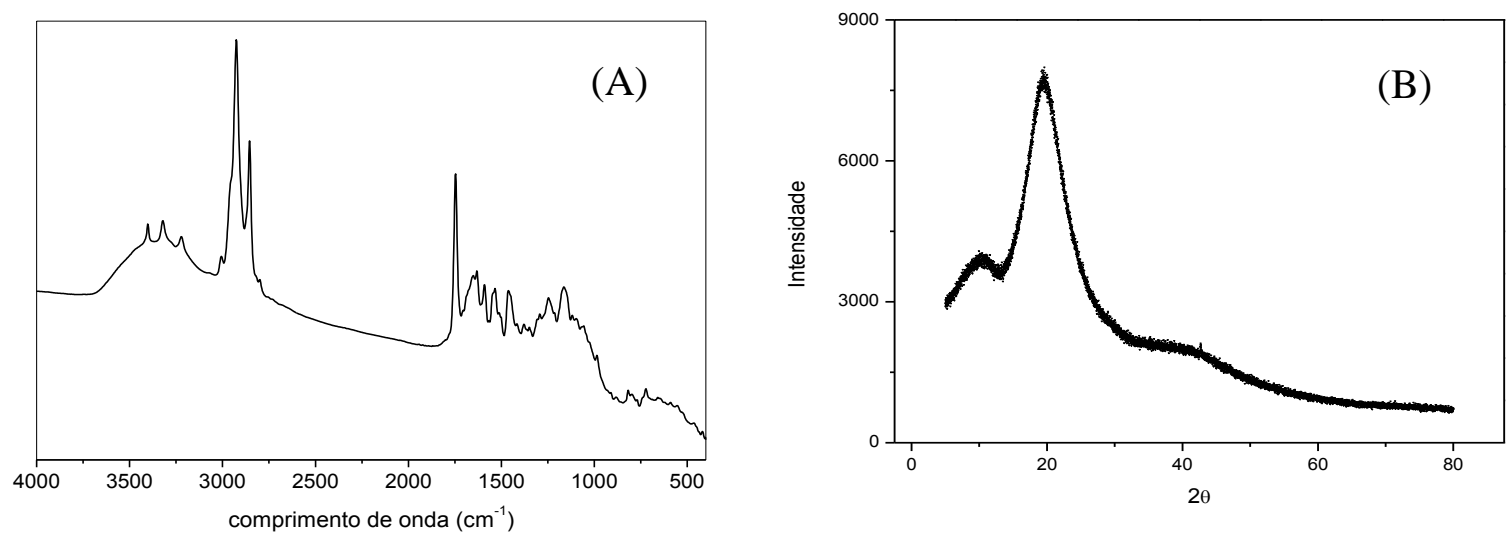

Figura 1 - Caracterização da semente de Moringa oleifera Lam: (A) Espectro de FTIR (B) Difratogramas de raios-X.

Os espectros de FTIR (Figura 1A) revelou a presença de vários grupos funcionais que indicam a natureza complexa da $\mathrm{MO}$ e presente papel importante no processo de adsorção. Foram encontrados a presença de grupos $\mathrm{OH}\left(3450-3350 \mathrm{~cm}^{-1}\right.$ ), os grupos de nitrogênio (aminas e iminas, 1745 e $1650 \mathrm{~cm}^{-1}$ ), ácidos carboxilicos $\left(1320\right.$ e $1246 \mathrm{~cm}^{-1}$ ) e gupos de Enxofre (tióis, as bandas de sinais fracos correspondente de ligação C-S, 530-975 $\mathrm{cm}^{-1}$ ). Três bandas sobrepostas à banda de $\mathrm{OH}\left(3400,3320\right.$ e $3225 \mathrm{~cm}^{-1}$ ) presente na amostra de sementes são devido ao alongamento da ligação NH (Reddy et al., 2011; Araújo et al., 2010). Pela Figura 1B, pode-se observar que devido ao alto teor de óleos e proteínas presentes na composição da semente (aproximadamente $69 \%$ em massa), o difratograma de raios-X mostra picos não-resolvidos, que indicam a predominância de material amorfo, característico de diversas biomassas (Araujo et al. 2010).

Na Figura 2 (A e B) pode-se observar que o material apresenta características morfológicas distribuídas com heterogeneidade e relativamente porosa. É visível a presença de deformações na superfície do tecido vegetal, contendo espaços disponíveis que possibilitam a condições favoráveis para adsorção. 

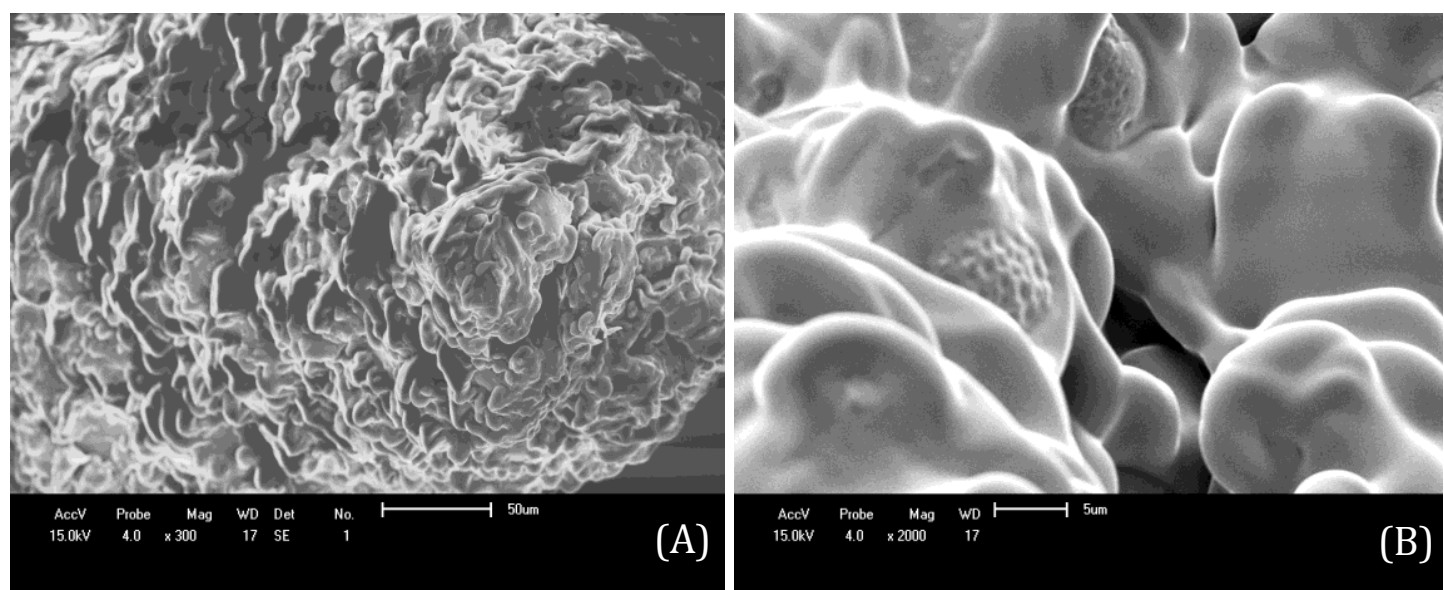

Figura 2 - Microscopia de Varredura Eletrônica Semente de Moringa oleifera. (A) magnitude 300X, (B) magnitude 2000X.

Um estudo preliminar do comportamento ácido-básico da superfície da semente $M$. oleifera, em meio aquoso, pode ser determinado através do PCZ. Pelo experimento, o patamar na região de $\mathrm{pH}$ 6,0 a 8,0 caracterizou o efeito tampão da superfície, o qual foi considerado o PCZ das sementes de $M$. oleifera. Em soluções com $\mathrm{pH}$ abaixo do PCZ, as sementes de $M$. oleifera apresentarão predominância de carga superficial positiva e em $\mathrm{pH}$ acima do PCZ, a carga superficial líquida será negativa.

Através da figura 4 (A e B), está demonstrado o comportamento da adsorção da atrazina em diferentes velocidades de agitação e valores de $\mathrm{pH}$.
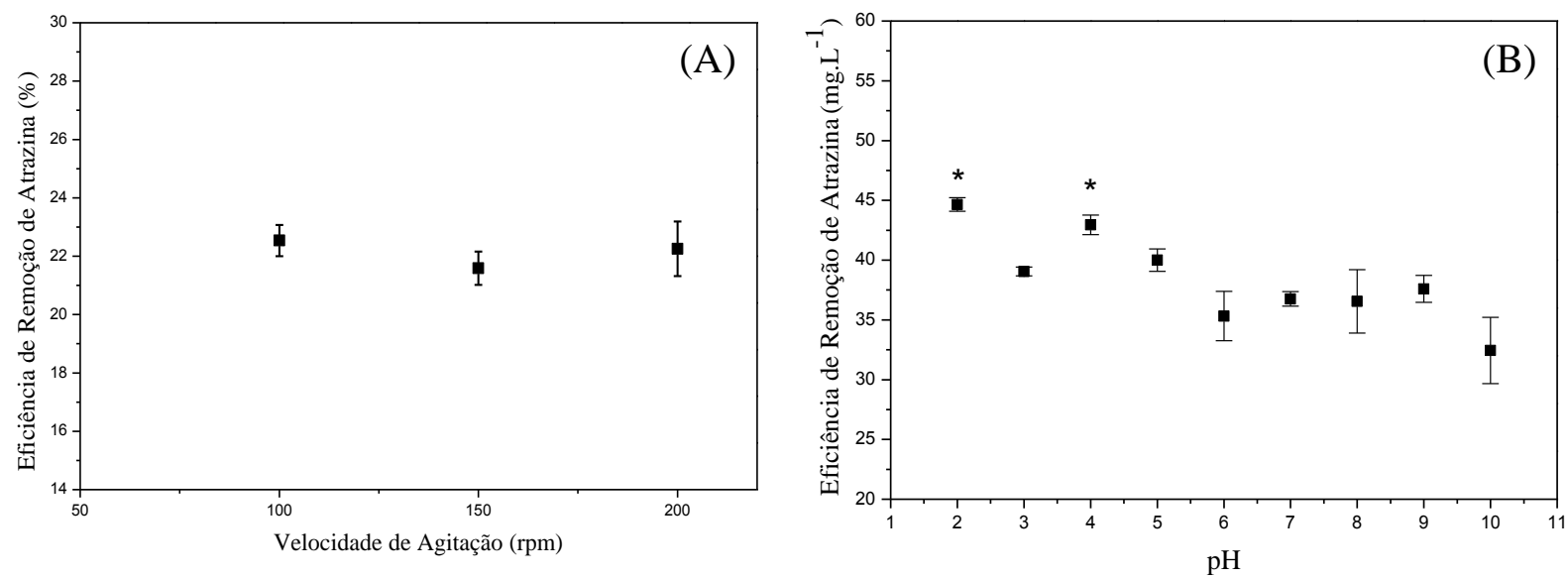

Figura 4 - (A) Efeito da velocidade de agitação; (B) Efeito do pH na eficiência de remoção de atrazina. ${ }^{*}$ Valores de $\mathrm{pH}$ não se diferenciam entre si, pelo teste de Tukey a 5\% de nível de significância. 
Através da Análise de Variância aplicado aos dados experimentais, verificou-se que o efeito da velocidade de agitação (Figura 4A) não é significativo, com um p-valor $=0,259$, assim optou-se para avaliar a cinética e as isotermas de adsorção pela velocidade de agitação de 100 rpm devido a menos custo energético. O efeito de $\mathrm{pH}$ é significativo (Figura 4B), com um p-valor $\leq 0,001$. Valores de $40 \%$ a $45 \%$ foram encontrados em $\mathrm{pH} 2-4$, não diferindo estatisticamente. A eficiência de adsorção de MO foi ligeiramente aumentada com uma diminuição no $\mathrm{pH}$. Isto pode ser atribuído à presença de íons hidrogênio a pH mais baixo, resultando em um aumento da captação de atrazina pela superficie do adsorvente com menor $\mathrm{pH}$.

Na figura 5, está demonstrado a eficiência de remoção de atrazina em relação a dosagem de adsorvente para diferentes temperaturas. Pode-se observar que há diferenças significativas p-valo $<0,001$ na adsorção da atrazina em dosagens de MO diferentes. Eficiências de remoção de atrazina entre $70 \%$ - $80 \%$ foram encontrados para 0,6 a $1,2 \mathrm{~g}$ de $\mathrm{MO}$, não diferindo estatisticamente. Nessas massas a temperatura não influenciou significativamente nos resultados. Sugere-se $0,6 \mathrm{~g}$ a dose adsorvente e uma temperatura de $25^{\circ} \mathrm{C}$.

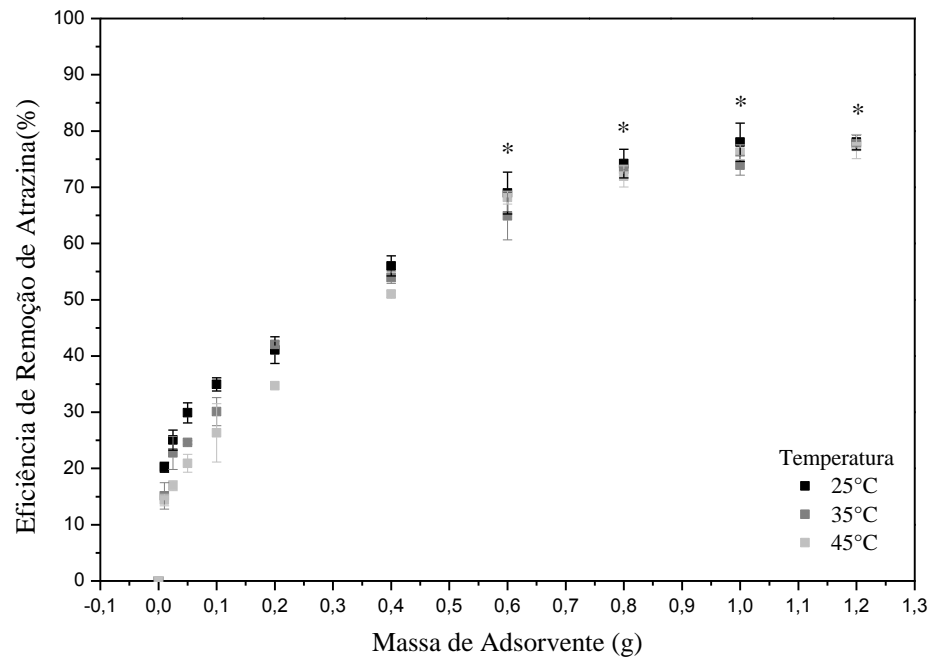

Figura 5 - Influência da temperatura e massa de adsorvente na eficiência de remoção de atrazina. ${ }^{*}$ Valores de massa de adsorvente nas temperaturas de $25^{\circ} \mathrm{C}$ a $45^{\circ} \mathrm{C}$ não se diferenciam entre si, pelo teste de Tukey a 5\% de nível de significância.

O ajuste dos dados experimentais aos modelos cinéticos de pseudo-primeira e pseudosegunda ordens, bem como os parâmetros obtidos a partir deste ajuste, são apresentados na Figura 6. Observa-se o equilíbrio da adsorção foi atingido a partir de 20 minutos, nesse período foi alcançado uma eficiência de remoção de atrazina aproximadamente de $78 \%$ e um qeq experimental $=0,352 \mathrm{mg} \mathrm{g}^{-1}$. O modelo cinético de pseudo-primeira-ordem foi o que melhor se ajustou aos dados experimentais. 


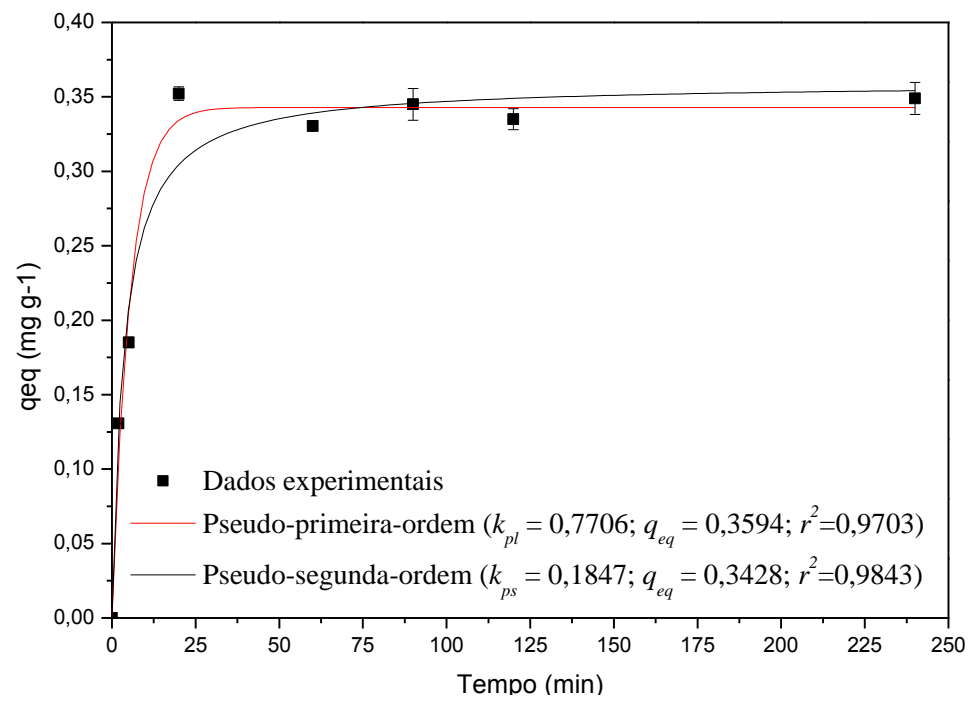

Figura 6 - Ajuste dos dados experimentais aos modelos cinéticos de pseudo-primeira e pseudosegunda ordens.

Na Tabela 1 está desmonstrado os modelos de Isoterma de Langmuir e Freundlich. O modelo que mais ajustou os dados experimentais foi modelo de Langmuir. Esse modelo sugere que a adsorção ocorre numa superfície homogênea por monocamada e sem interacção entre as moléculas adsorvidas.

Tabela 1 - Constantes das Isotermas de Langmuir e Freundlich para a adsorção de atrazina em sementes de Moringa oleifera.

\begin{tabular}{cccccc}
\hline & Constantes de Lagmuir & \multicolumn{3}{c}{ Constantes de Freundlich } \\
\hline $\begin{array}{c}\mathbf{q}_{\text {eq }} \\
(\mathbf{m g} \mathbf{g}-\mathbf{1})\end{array}$ & $\begin{array}{c}\mathbf{b}_{\mathbf{L}} \\
(\mathbf{L m g - 1 )}\end{array}$ & $\mathbf{R}^{\mathbf{2}}$ & $\mathbf{n}_{\mathbf{f}}$ & $\begin{array}{c}\mathbf{k}_{\mathbf{f}} \\
(\mathbf{m g} \mathbf{g}-\mathbf{1})\end{array}$ & $\mathbf{R}^{\mathbf{2}}$ \\
\hline 0,578 & 0,203 & 0,971 & 0,55 & 0,108 & 0,934 \\
\hline
\end{tabular}

\section{CONCLUSÕES}

A semente de Moringa oleifera Lam apresentou-se como um adsorvente em potencial para a remoção de atrazina, com uma percentagem de remoção variando de $70 \%$ a $80 \%$. Pela caracterização de FTIR, DRX e MEV, observou-se que MO apresenta grupos funcionais e uma morfologia que podem promover a adsorção de atrazina. Verifiou-se pela análise de PCZ e estudo do efeito de $\mathrm{pH}$ que a adsorção da atrazina é melhorada em $\mathrm{pH}$ ácido. Uma vez que a semente de Moringa é um subproduto e não tenha sido previamente submetido a um tratamento químico ou físico, pode ser considerada como uma alternativa eficiente e de baixo custo para remoção de Atrazina. 


\section{9 a 22 de outubro de 2014 \\ Florianópolis/SC}

\section{REFERÊNCIAS}

AKHTAR, M., HASANY, S. M., BHANGER, M. I., IQBAL, S. Low Cost Sorbents for the Removal of Methyl Parathion Pesticide from Aqueous Solutions, Chemosphere, v. 66, p.1829-1838, 2007.

ALMEIDA, I. L. S. Avaliação da capacidade de Adsorção da Torta de Moringa Oleifera para BTEX em amostras aquosas. Dissertação (Mestrado em Química) - Universidade Federal de Uberlândia, Minas Gerais, 2010.

ALVES, V. N. and N. M. M. COELHO Selective extraction and preconcentration of chromium using Moringa oleifera husks as biosorbent and flame atomic absorption spectrometry. Microchemical Journal v. 109 p. 16-22,2013.

AMAGLOH, F. K.; BENANG, A. Effectiveness of Moringa oleifera seed as coagulant for water purification. African Journal of Agricultural Research, v. 4, n. 1, p. 119-123, 2009.

ARAUJO, C. S. T., V. N. ALVES, H. C. REZENDE, I. L. S. ALMEIDA, R. M. N. DE ASSUNCAO, C. R. T. TARLEY, M. G. SEGATELLI and N. M. M. COELHO Characterization and use of Moringa oleifera seeds as biosorbent for removing metal ions from aqueous effluents. Water Sci Technol v. 62, p. 2198-2203,2010.

AVILA, L. G.; LEITE, S. B.; DICK, D. P. Formulações de atrazina em xerogéis: Síntese E Caracterização. Quim. Nova, v. 32, n. 7, p.1727-1733, 2009.

BEZERRA, A. M. E.; MOMENTÉ, V. G.; MEDEIROS FILHO, S. Germinação de sementes e desenvolvimento de plântulas de moringa (Moringa oleifera Lam.) em função do peso da semente e do tipo de substrato. Horticultura Brasileira, Brasília, v.22, n.2, p.295-299, abril-junho 2004.

BORTOLUZZI E. C. et al. Investigation of the occurrence of pesticide residues in rural wells and surface water following application to tobacco. Quim. Nova. v. 30, n. 8, p. 1872-1876, 2007.

BRASIL. Ministério da Saúde, Portaria no 2.914, de 12 de dezembro de 2011. Dispõe sobre os procedimentos de controle e de vigilância da qualidade da água para consumo humano e seu padrão de potabilidade. Disponível

em: http://bvsms.saude.gov.br/bvs/saudelegis/gm/2011/prt2914_12_12_2011.html

CUNHA, G. C.; CARDOSO, M. S.; ROMÃO, L. P. C.; DOMINGOS, I. G. R. Fitorremediação dos trihalometanos pela Moringa oleifera In: 34 reunião anual Sociedade Brasileira de Química. 2011. Disponivel em: http://sec.sbq.org.br/cdrom/34ra/resumos/T1875-1.pdfd Acesso: 20/04/2014.

EUROPEAN COMMISSION (2008). Directive 2008/105/EC. Disponível em: http://ec.europa.eu/environment/water/water-framework/priority_substances.htm (accesso 12.04.14.).

KWAAMBWA, H., MAIKOKERA, R. Infrared and circular dichroism spectroscopic characterisation of secondary structure components of a water tratment coagulnt protein extracted from Moringa oleifera seeds. Colloids and Surfaces B: Biointerfaces, v. 64, p. 118-125, 2008.

OKUDA, T.; BAES, A. U.; NISHIJIMA, W.; OKADA, M., Isolation and characterization of coagulant extracted from Moringa oleifera seed by salt solution, Water Research, v. 35 n. 2, p. 405-410, 2001.

REDDY, D. H. K., D. K. V. RAMANA, K. SESHAIAH and A. V. R. REDDY Biosorption of $\mathrm{Ni}$ (II) from aqueous phase by Moringa oleifera bark, a low cost biosorbent. Desalination v. 268 p. 150$157,2011$.

REGALBUTO, J. R.; ROBLES, J.; The engineering of Pt/Carbon Catalyst Preparation, University of Illinois: Chicago, 2004.

US EPA Environmental Protection Agency; Draft final list of recommendation for chemicals in the National Survey for Pesticides in Groudwate,. Chem. Regul. Rep. v. 9, p. 1033, 1985. 